\title{
Research
}

\section{Parental reasons for non-uptake of influenza vaccination in young at-risk groups:}

\author{
a qualitative study
}

\begin{abstract}
\section{Background}

Uptake rates of influenza vaccination in young at-risk groups in primary care (UK) are known to be poor.

\section{Aim}

To explore parental reasons for non-uptake of influenza vaccination in young at-risk groups. The study hypothesis was that exploration of parental reasons for non-uptake may reveal important barriers to an effective influenza vaccination programme.
\end{abstract}

\section{Design and setting}

Thematic analysis of a questionnaire survey with interview follow-up at a single general practice in Inverness, Scotland.

\section{Method}

Parents of children identified as being in an atrisk group for influenza vaccination but who had not received vaccination were sent questionnaires and offered the opportunity to take part in a follow-up interview.

\section{Results}

Several key themes emerged, including uncertainty about the indication for vaccination, issues of choice, challenges with access, lack of parental priority, and issues relating to health beliefs.

\section{Conclusion}

Any attempt to improve the vaccination rate needs to address the range of decision-making processes undertaken by parents and children. Better and more tailored information and educational delivery to parents, patients, and healthcare providers may lead to an increase in the rates of influenza vaccination uptake in atrisk children. Access is a barrier described by some parents.

\section{Keywords}

children; influenza; population at risk; primary care; vaccination.

\section{INTRODUCTION}

Influenza vaccination rates in at-risk children are low across the UK. ${ }^{1-3}$ Analysis of the 2008/2009 uptake figures in England suggests that $23.8 \%$ of those aged $2-16$ years in an at-risk group were vaccinated, compared with $50.2 \%$ of those in an at-risk group aged $16-65$ years, and $74.1 \%$ of those aged $\geq 65$ years. ${ }^{3}$ The vaccination programme for children laged less than 16 years) in the UK is targeted at specific at-risk groups by the Department of Health. ${ }^{4}$ This study aimed to develop a better understanding of the reasons for poor uptake lin those aged 2-16 years in an at-risk group), in order to inform ways of improving rates of vaccination. The importance of this is that there is a greater risk of those aged under 16 years in an atrisk group developing complications from influenza compared with those not in an atrisk group. ${ }^{5}$

Given the age range of the target group (those in an at-risk group aged $2-16$ years old), it was reasonable to assume that parental influences may play a key role in whether or not a child is vaccinated. It was therefore decided to ask the question: what are the parental reasons for non-uptake of influenza vaccination in young at-risk groups?'.

A literature search using the keywords listed in Box 1 failed to identify previous studies undertaken in the UK primary care setting to investigate parental reasons for poor uptake of influenza vaccination among at-risk children.

R Sampson, MRCGP, GP principal, Cairn Medical Practice and associate advisor North Deanery Scotland, Inverness. R MacVicar, FRCGP, GP principal, Cairn Medical Practice and director of Postgraduate GP Education, North Deanery Scotland, Inverness. L Wong, MRCP, MRCGP, sessional GP, Albyn Medical Practice, Aberdeen Address for correspondence

Dr Rod Sampson, Cairn Medical Practice,
METHOD

Using the Vision-INPS audit tool, the children eligible for immunisation in the 2007/2008 influenza vaccination campaign were identified from the patient database of a single medical practice in Inverness in the Highlands of Scotland (total practice population 9700). The search was based on the Department of Health inclusion criteria for recommending influenza vaccination of young at-risk groups (Box 2).

The parents of children who were registered with the practice and who met the inclusion criteria (Box 3) were invited in November 2008 to take part in the study. A time frame was chosen to reflect the entirety of the influenza season 11 September 2007 to 31 March 2008), to gain as accurate a picture as possible. An age range of 2-16years was chosen, to be consistent with both the Vision-INPS audit tool and the age range used by the UK Department of Health in its monitoring of uptake rates.

The parents of those children that met the inclusion criteria (the participants in the studyl were sent 'Participant Study Pack 1', which contained a letter of invitation, a research participant information sheet, a consent form, and questionnaire 1 lavailable from the authors). Non-responders at 4 weeks were excluded from the study. Questionnaire 1 ended with an invitation to take part in the second part in the study. Responders indicating they were unaware that influenza vaccination was recommended for their child were excluded

Ardlarich, 15 Culduthel Road, Inverness, IV2 4AG.

E-mail: rod.sampsonanhs.net

Submitted: 29 January 2011; Editor's response: 9 March 2011; final acceptance: 5 May 2011 (cBritish Journal of General Practice

This is the full-length article (published online 27 Jun 2011) of an abridged version published in print. Cite this article as: Br J Gen Pract 2011; DOI: 10.3399/bjgp11X583155. 


\section{How this fits in}

The UK Department of Health has clear recommendations for influenza vaccination of 'at-risk' groups. Despite this, uptake rates of influenza vaccination in children and young people (less than 16 years old) in 'at-risk' groups in primary care is less than for other groups. A majority of parents lo those aged $<16$ years considered to be in an at-risk groupl that responded to a questionnaire in this study were not aware that their child was recommended to receive influenza vaccination. Any vaccination programme needs to address this issue and also the range of decisionmaking processes undertaken by parents and their children.

from further participation in the study. Those indicating a willingness to take part in the second part of the study received 'Participant Study Pack 2', which contained a second consent form and questionnaire 2 (available from the authors). Questionnaire 2 could be completed either in writing, or in a telephone interview or a face-to-face interview, according to participant preference. A variety of modes of response was offered to encourage participation. Written responses to both questionnaires, along with audiorecording of interviews, were transcribed by administrative staff in

\section{Box 2. Clinical risk groups}

\section{Chronic respiratory disease}

Chronic obstructive pulmonary disease (COPD) including chronic bronchitis and emphysema; bronchiectasis, cystic fibrosis, interstitial lung fibrosis, pneumoconiosis, and bronchopulmonary dysplasia (BPD), chronic respiratory disease and asthma that requires continuous or repeated use of inhaled or systemic steroids or with previous exacerbations requiring hospital admission

\section{Chronic heart disease}

Congenital heart disease, hypertension with cardiac complications, chronic heart failure. Individuals requiring regular medication and/or follow-up for ischaemic heart disease

\section{Chronic renal disease}

Chronic renal failure, nephrotic syndrome, renal transplantation

\section{Chronic liver disease}

Cirrhosis, biliary atresia, chronic hepatitis

\section{Chronic neurological disease}

Stroke, transient ischaemic attacks (TIAs), multiple sclerosis and related conditions, hereditary and degenerative disease of the central nervous system

\section{Diabetes mellitus}

Type 1 diabetes, type 2 diabetes requiring insulin or oral hypoglycaemic drugs, diet-controlled diabetes

\section{Immunosuppression}

Immunosuppression due to disease or treatment. Patients undergoing chemotherapy leading to immunosuppression. Asplenia or splenic dysfunction, HIV infection, individuals treated with, or likely to be treated with, systemic steroids for more than a month at a dose equivalent to prednisolone $\geq 20 \mathrm{mg} /$ day (any age), or for children under $20 \mathrm{~kg}$, a dose of $1 \mathrm{mg} / \mathrm{kg} / \mathrm{day}$ the medical practice onto a Microsoft Excel spreadsheet. The text was analysed manually by two of the authors independently, to capture and code the themes that arose. No qualitative analysis software package was felt by the authors to be necessary. A subsequent joint exercise was undertaken to compare the themes that arose from this process of independent analysis, resulting in an enhanced set of themes. ${ }^{6}$

\section{RESULTS}

Details of the process and numbers involved in the study are illustrated in Figure 1. A total of 53 participants were invited to take part in the study; 15 participants were nonresponders' at 4 weeks and so were excluded according to the study design. Of the 38 who responded to the invitation and agreed to participate in the study, 22 were not aware that the influenza vaccination was recommended for their child, and, according to the study design, took no further part. The remaining 16 completed the initial questionnaire but only nine of this number consented to provide further information. Of these, five preferred to respond in writing and two preferred a telephone interview. Two chose not to respond further. No participant expressed a preference for face-to-face interview. The final number of participants in part 2 of the study was therefore seven.

Five main themes evolved from analysis of the data (Box 4).

\section{Uncertainty about indication for vaccination}

Some parents described issues that would be recognised by health professionals as contraindications to the vaccine, notably egg allergy. However, despite the fact that all children in this study fell within the group for whom seasonal influenza vaccination is indicated, a much greater proportion of parents expressed doubt, scepticism, or a lack of knowledge about the relevance of the vaccination for their child:

[Child's] asthma had seemed to be "dormant" for several years so we didn't think a flu jab was necessary. Also, we thought as her asthma is quite mild she wasn't high risk.' (Parent [P] 1)

I have asked if [child] was able and told only if she was hospitalised at some point with asthma. I feel myself she would not benefit from it as her asthma is mild.' (P31)

Unconvinced that the vaccine will fight the 


\section{Box 3. Inclusion and exclusion criteria}

\section{Inclusion criteria}

Parents of children eligible for vaccination aged 2-16 years, who did not receive influenza vaccination over a 7-month period (1 September 2007 to 31 March 2008).

\section{Exclusion criteria}

Eligible children who were no longer registered in the practice on or before 31 March 2008. Eligible children who turned 17 years on or before 31 March 2008. Those parents who did not consent to taking part in the study.

\section{strain of flu that's around.' (P19)}

These comments reveal a need for parent education regarding vaccine indication for their children, and also the need for effective communication about the recommendation for vaccination and risks of non-vaccination between the healthcare provider and parent.

\section{Issues of choice}

Issues of choice were strongly represented in the data. While the choice described was usually that of the parent, choice for the doctors involved in the care of the child, and
Figure 1. Summary flowchart of protocol with participant numbers in brackets and timescale in italics. indeed those of the children themselves, were taken into account in parental decisions about vaccination:

We chose not to - this was due to our understanding that there are many different flu viruses and that the jab only protects against the "most likely".' (P19)

'Has been diabetic since age seven, is now 16 therefore would have had nine unnecessary injections.' (P28)

After consulting with [child's] doctor about this vaccine in relation to [child's] egg allergy, the doctor in turn consulted a paediatrician at [hospital] Iwhere the child received his first MMR [measles, mumps and rubella] jab last year with no obvious ill effects) who advised against him receiving the vaccine in 2007. Even if the doc [paediatrician] had advised going ahead with the flu vaccine, I don't think I would have, for various reasons!' (P35)

'She didn't want the flu jab as she is afraid of needles.' (P43)

While choice will always play a key role in decisions of parents as to whether to vaccinate their children, there is a key role for healthcare providers in ensuring that the choices that are made are informed ones.

\section{Challenges with access}

Analysis of the data revealed several described barriers to accessing vaccination. These included a lack of a personal invitation from the practice, difficulties gaining an appointment, and the challenges of intercurrent illnesses compounding appointment difficulties:

'He wasn't using his steroid inhaler all the time (mainly during hay fever season) and as he wasn't asked to come we were unsure of his eligibility for the vaccine.' (P21)

I made an appointment for [child] to get her flu jab, the clinic was busy and it was well into November before I could get an appointment for her. By which time she was unwell with chest infections, or if not had temperatures. [Child] did actually get her flu jab last winter but it was actually February 2008 before she was well enough to have it.' (P33)

At present, responsibility for invitation for vaccination lies with individual GP practices, without regional or national coordination. There is likely, therefore, to be wide variation in how this invitation process is managed 


\section{Box 4. Themes}

- Uncertainty about indication for vaccination

- Issues of choice

- Challenges with access

- Lack of parental priority

- Issues relating to health beliefs and delivered. Given the age range (2-16 years) of this group, specific access arrangements may need to take into account the timing of nursery and school compared with simply setting up a 'one size fits all'-type influenza clinic.

\section{Lack of parental priority}

Some parents were very frank about the lack of priority they placed on ensuring that their child was protected by influenza vaccination, despite apparently being aware of the potential benefits:

Not really, I actually meant to but did not get round to it, to be honest!' (P46)

Raising awareness of the importance of influenza vaccination in at-risk groups and the potential consequences of nonvaccination may well influence the priority placed by parents on ensuring that their children are vaccinated.

\section{Issues relating to health beliefs}

In contrast to the parents for whom a lack of vaccination represented an apparent lack of parental priority, there was a group of parents who seem to have thought deeply about the issues of vaccination, and their decisions were based on the beliefs that they hold about vaccination. These beliefs related to both the influenza vaccination in particular and vaccination in general, and often related to their experience of media reporting of vaccination 'scares':

'I read the leaflet about it (NHS one I think) and tried to make a balanced judgement based on that info and my own knowledge of my son's health - I chose not to have it. At the end of the day, GPs are always I think going to be faced with parents who for whatever reasons think they know best about their child - whether rightly or wrongly! Media scares about vaccines are hard to shake off, and I think have an impact on one's perception of vaccines in general. So while there's not to my knowledge been anything scary said about the flu jab per se, I still feel a bit uneasy about another vaccine for small bodies. I suppose promoting the positives might help convince some people.' (P35)

Some parents expressed difficulty in accepting that the vaccination is as important for children with chronic health problems as it is for older people:

'The focus is more on older ... it's difficult to imagine a child getting the flu and being very ill.' (P19)
He has never shown any flu symptoms and we feel that he is young to be starting to give him this kind of injection. Also, we have had experience of older members of the family contracting the flu following the injection and we did not want our son to be at risk.' (P54)

Concerns were also expressed about the vaccine itself - whether in some way it will make an influenza-like illness more likely or whether it could have an adverse impact on the development of their child's immune system:

'I suppose with any vaccine whether it has short- or long-term side effects that might not yet be evident, whether it's absolutely necessary, or whether it's better to let their immune systems build up their own defences. Whether it may be one vaccine too many? Vaccine overload, as you hear spoken about in the media - are we doing the right thing or not with all these vaccines?' (P35)

While no clear evidence exists to support the health beliefs described, they can be understood in a climate of negative media reporting of both influenza vaccination, and vaccination in general. Anecdotal evidence is often more powerful than established evidence; for example, although influenza vaccine is an inactivated vaccine, and it is not possible to contract influenza from the vaccination, this firm belief led one family not to vaccinate their child.

\section{DISCUSSION}

\section{Summary}

Although several key themes arose further in the responses from parents of children for whom the vaccination was recommended, more than half of participants in this study were not aware the influenza vaccination was recommended for their child (22 out of 38 participants). Therefore, even if all the participants in this study who were aware that their child needed the influenza vaccination were vaccinated, at best less than half of the desired coverage in this population could have been achieved.

For those parents who were aware of the recommendation that their child be vaccinated, the responses suggest that the degree of consideration given to the issue varies widely, from a lack of engagement, to decisions made on flimsy evidence, to complex and agonised decision making. Support for parents and children in coming to a decision to vaccinate their child against influenza needs to take into account this range of approaches to include better and 
tailored provision of information, and appropriate use of the media to counter misinformation about the effect and role of vaccination, as well as to provide positive information.

The study also emphasises the role for GPs and primary care professionals in improving the vaccination rate. Parents often expect an invitation to attend for vaccination, and problems with access were described. To improve vaccination, general practices will need to address these issues to improve vaccination rates. Parents also reported experience of information emanating from the health service that may betray some ambivalence from health professionals about the benefit of influenza vaccination for children, and this will form the basis of a future study.

\section{Strengths and limitations}

The authors believe this is the first study done in the UK primary care setting to investigate parental reasons for poor influenza immunisation uptake among atrisk children. There are several potential limitations of the study. The research was carried out in one practice by researchers who were the GPs of the participants. As a result, there is potential for some researcher bias and also a potential for participants to be less open than they would be to an independent researcher. The sample size of seven participants is small. This raises some uncertainty about how applicable the findings are to the wider population of children that are eligible for influenza vaccination. The initial search carried out to identify the children of participants was dependent on the accuracy of coding and robustness of the coding system of the medical practice. Inverness is one of the UK's youngest cities Igaining city status in the year 2000), and the demographics of practice populations in Inverness may not be representative of the wider UK population. The study site is an urban practice and the results may not be applicable to rural or more deprived innercity populations. Readers may wish to explore whether the issues raised within the study also impact on the uptake of vaccination where they practice.

\section{Comparison with existing literature}

Previous literature has highlighted the inadequacy of delivery of influenza vaccine to children in at-risk groups. ${ }^{3,5}$ Several factors are known to influence childhood influenza vaccination, including the key parental belief that a child may not be at risk from influenza. ${ }^{7}$ Primary care has a key role in education about, and delivery of, the influenza vaccine to those in the young atrisk groups. Previous literature has indicated the importance of vaccine recommendation by a GP as being influential in determining whether vaccination is accepted.8-12 The potential for a strong recommendation from a GP to influence uncertainty about the indication for the vaccination, issues of choice, and health beliefs is illustrated in the key themes that arose in the present study. Health beliefs surrounding the vaccination are important issues in influencing compliance. This study has confirmed previous findings that parents are not convinced about the seriousness of influenza in children, ${ }^{8}$ and that positive correlations with immunisation rates include perception of the severity of the child's condition and negative correlations with the risk of vaccine side effects. ${ }^{13}$

\section{Implications for practice and research}

There is ongoing debate regarding the merits of universal vaccination for all children, not only in order to reduce morbidity among children, but also to reduce levels of circulating virus in the wider community. At present in the UK, there are no plans to change current immunisation recommendations. ${ }^{?}$

Any local or national programme promoting vaccination in young at-risk groups must take account of the potential barriers to vaccination highlighted by the study. Better and more targeted information and educational delivery to parents, patients, and healthcare providers in the context of the issue being given higher national and local priority, may lead to an increase in the rates of influenza vaccination uptake in atrisk children. Given that 22 out of 38 participants who agreed to take part in the study were not aware the influenza vaccination was recommended for their child, GP practices have a key role in the identification of those eligible for influenza vaccination and invitation for vaccination, including follow-up on non-attenders.

One reason for parents not being aware that their child may benefit from vaccination may be that healthcare professionals are unaware of, or unconvinced by the potential benefits of immunisation in at-risk individuals aged less than 16 years. Given the importance of the role of the GP in influencing positive compliance with influenza vaccination uptake in young atrisk groups, further study is planned to explore GP attitudes to vaccination in this important cohort. 


\section{REFERENCES}

1. Joseph C, Goddard N, Gelb D. Influenza vaccine uptake and distribution in England and Wales using data from the General Practice Research Database, 1989/90-2003/04. J Public Health 2005; 27(4): 371-377.

2. Chantler T, Pace D, Wright A, et al. Uptake and acceptability of influenza vaccination in day nursery children. Community Pract 2007; 80(12): 32-36.

3. Influenza Immunisation Uptake Monitoring Programme. Influenza vaccine uptake among the 65 years and over and under 65 years at risk in England winter season 2008-09.

http://wnw.dh.gov.uk/prod_consum_dh/groups/dh_digitalassets/documents/digi talasset/dh_116624.pdf laccessed 7 Jun 2011).

4. Department of Health. Immunisation against infectious disease - 'The Green Book'. London: Department of Health, 2007.

http://wnw.dh.gov.uk/en/Publichealth/Healthprotection/Immunisation/Greenboo k/DH_4097254 laccessed 13 Jun 2011).

5. Esposito S, Marchisio $P$, Droghetti $R$, et al. Influenza vaccination coverage among children with high-risk medical conditions. Vaccine 2006; 24(24): 5251-5255.

6. Charmaz K. Constructing grounded theory - a practical guide through qualitative analysis. London: Sage Publications, 2006.

7. Grant VJ, Le Saux N, Plint AC, et al. Factors influencing childhood influenza immunization. CMAJ 2003; 168(1): 39-41.

8. Chantler T, Pace D, Wright A, et al. Uptake and acceptability of influenza vaccination in day nursery children. Community Pract 2007; 80(12): 32-36.

9. Blank PR, Schwenkglenks M, Szucs TD. Vaccination coverage rates in eleven European countries during two consecutive influenza seasons. J Infect 2009; 58(6): 446-458.

10. Nowalk MP, Lin CJ, Zimmerman RK, et al. Changes in parents perceptions of infant influenza vaccination over 2 years. J Natl Med Assoc 2007; 99(6): 636-641.

11. Lin CJ, Nowalk MP, Zimmerman RK, et al. Beliefs and attitudes about influenza immunization among parents of children with chronic medical conditions over a two-year period. J Urban Health 2006; 83(5): 874-883.

12. Gnanasekaran SK, Finkelstein JA, Hohman K, et al. Parental perspectives on influenza vaccination among children with asthma. Public Health Rep 2006; 121(2): 181-188

13. Szilagyi PG, Rodewald LE, Savageau J, et al. Improving flu vaccination rates in children with asthma: a test of a computerised reminder system and an analysis of factors predicting vaccination compliance. Pediatrics 1992; 90(6): 871-875. 